The Astrophysical Journal, 560:110-118, 2001 October 10

(C) 2001. The American Astronomical Society. All rights reserved. Printed in U.S.A.

\title{
H I GAS IN THE ENVIRONMENT OF THE SEYFERT GALAXIES NGC 6764 AND MARKARIAN 1126
}

\author{
ERIC M. WILCOTS \\ Department of Astronomy, University of Wisconsin, 475 North Charter Street, Madison, WI 53706; ewilcots@astro.wisc.edu \\ MARgaret C. TurnbUll \\ Steward Observatory, 933 North Cherry Avenue, Tucson, AZ 85721; turnbull@as.arizona.edu \\ AND \\ ELIAS BRINKS \\ Universidad de Guanajuato, Departamento de Astronomía, Apartado Postal 144, Guanajuato, C.P. 36000, Mexico \\ Received 2001 February 14; accepted 2001 June 19
}

\begin{abstract}
We present the results of a $21 \mathrm{~cm}$ line study of the environment of two Seyfert galaxies: NGC 6764 and Mrk 1126. Both galaxies are embedded in large and slightly distorted gaseous disks that extend 2-4 times farther than their optical diameters. The asymmetry of the $\mathrm{H}$ I distribution and profile is indicative of a weak perturbation, although one that is not strong enough to disturb either the gas or the stars in the inner parts of the galaxies. Whereas Mrk 1126 does have a nearby companion, MCG $-02-58-017$, the gravitational interaction between the two galaxies is quite weak, and it is unlikely that MCG $-02-58-017$ is responsible for driving the nuclear activity in Mrk 1126. NGC 6764 has no companions within a radius of $160 \mathrm{kpc}$. We conclude that the nuclear activity in NGC 6764 and Mrk 1126 is unrelated to the galaxies' immediate environment.

Subject headings: galaxies: individual (NGC 6764, Markarian 1126) - galaxies: interactions galaxies: ISM - galaxies: Seyfert
\end{abstract}

\section{INTRODUCTION}

Even though there has been no lack of effort to identify external triggers to nuclear activity in galaxies, the results have been mixed. Studies of optical plates suggest that Seyfert activity is more prevalent among interacting galaxies, or at least that Seyfert galaxies tend to reside in regions of higher galactic density than non-Seyfert galaxies (Adams 1977; Dahari 1984). More recent CCD studies dispute this claim, showing evidence that there is no distinguishable difference between the environments of Seyfert and normal field galaxies (Fuentes-Williams \& Stocke 1988; Laurikainen \& Salo 1995; De Robertis, Yee, \& Hayhoe 1998; Virani, De Robertis, \& Vandalfsen 2000). There is, however, some support for the notion that the environment of Seyfert 2 galaxies is richer than that of Seyfert 1 and field galaxies (e.g., De Robertis et al. 1998; Virani et al. 2000). Direct evidence that Seyfert galaxies are interacting systems is quite scarce. An obvious example is Mrk 348, a galaxy whose $\mathrm{H}$ I distribution reaches a diameter that is some 7 times that of its stellar distribution (Simkin et al. 1987). These detailed H I observations revealed Mrk 348 to be an interacting system. Similarly, H I observations of NGC 3227 show it to be strongly interacting with a nearby elliptical, NGC 3226 (Mundell et al. 1995). But these cases are the exception and not the rule. With this backdrop, De Robertis et al. (1998) concluded that Seyfert activity is not driven by major interactions in most galaxies, but may possibly be associated with minor mergers - the accretion of small clumps of gas or dwarf galaxies amounting to no more than $10 \%$ of the mass of the Seyfert.

Minor mergers have been equally difficult to observe, although Haynes et al. (2000) have detected counterrotating $\mathrm{H} \mathrm{I}$ in a number of early-type spirals, and a number of $\mathrm{H} \mathrm{I}$ observations over the past decade have revealed the pres- ence of small $\mathrm{H}$ I clouds around larger, usually late-type galaxies (e.g., Wilcots, Lehman, \& Miller 1996). Moreover, several galaxies appear to be accreting neighboring gas clouds (e.g., Phookun, Vogel, \& Mundy 1993; Wilcots et al. 1996; Pisano, Wilcots, \& Elmegreen 1998). The connection between these clouds and the characteristics of the primary galaxy, however, remains unclear, even though numerical studies indicate that the accretion of $10^{7} M_{\odot}$ worth of gas would have dramatic effects on the primary galaxy (Hernquist \& Mihos 1995; Haynes et al. 2000). Seyfert activity could be among those effects, but to date there is no Seyfert for which there is unambiguous evidence of a recent accretion event.

Given this uncertainty in the connection between a Seyfert's immediate environment and its nuclear activity, we set out to map the neutral hydrogen distribution around a small sample of Seyfert galaxies to assess the extent to which the gaseous environment of these particular galaxies influences their nuclear activity. Our initial goal was to obtain direct evidence that the galaxy is participating in an interaction or has recently participated in an interaction. Here, we present the results for two galaxies residing in different environments. Mrk 1126 resides in a small group of galaxies (Garcia 1993), while NGC 6764 is relatively isolated. We list some of the salient features of both objects in Table 1 .

We briefly describe our observations and data reduction in the next section. We detail the results for NGC 6764 in $\S 3$ and for Mrk 1126 in $\S 4$. We place this study into the context of the connection between galaxy properties and their immediate environment in $\S 5$.

\section{OBSERVATIONS AND REDUCTIONS}

Both NGC 6764 and Mrk 1126 were observed in the D 
TABLE 1

BASIC PROPERTIES

\begin{tabular}{|c|c|c|}
\hline Property & NGC 6764 & Mrk 1126 \\
\hline$\alpha_{1950}$ & 190816.4 & 230047.8 \\
\hline$\delta_{1950} \ldots \ldots \ldots \ldots \ldots \ldots \ldots$ & +505600 & -125507 \\
\hline Hubble type ${ }^{\mathrm{a}} . . . . . . .$. & $\mathrm{SB}(\mathrm{s}) \mathrm{bc}$ & $(\mathrm{R}) \mathrm{SB}(\mathrm{r}) \mathrm{a}$ \\
\hline$m_{B}^{\mathrm{a}} \ldots \ldots \ldots \ldots \ldots \ldots \ldots \ldots$ & 12.6 & 14.0 \\
\hline$L_{B_{\odot}} \ldots \ldots \ldots \ldots \ldots \ldots \ldots$ & $1.5 \times 10^{10}$ & $6.3 \times 10^{9}$ \\
\hline $\mathrm{Size}_{\text {optical }}(\operatorname{arcsec})^{\mathrm{a}} \ldots \ldots$ & $2.3 \times 1.2$ & $1.8 \times 1.8$ \\
\hline Distance $^{\mathrm{b}}(\mathrm{Mpc}) \ldots \ldots$ & 37 & 49 \\
\hline$M_{\mathrm{H}_{\mathrm{I}}}^{\mathrm{c}}\left(M_{\odot}\right) \ldots \ldots \ldots \ldots \ldots$ & $(7 \pm 1) \times 10^{9}$ & $(7 \pm 1) \times 10^{9}$ \\
\hline
\end{tabular}

NotE.- Units of right ascension are hours, minutes, and seconds, and units of declination are degrees, arcminutes, and arcseconds.

${ }^{a}$ From NED.

b Assuming $H_{0}=65 \mathrm{~km} \mathrm{~s}^{-1} \mathrm{Mpc}^{-1}$.

${ }^{c}$ This paper.

configuration of the Very Large Array ${ }^{1}$ on 1995 April 1. The observational parameters are listed in Table 2. We observed each galaxy in 4IF mode, choosing to split the intermediate frequency (IF) pairs in order to maximize our velocity coverage while maintaining modest $\left(20 \mathrm{~km} \mathrm{~s}^{-1}\right)$ velocity resolution. Periodic observations of $0134+329$ (amplitude and bandpass) and $1922+333$ and 2243-123 (phase) were used for calibration. We flagged widely discrepant visibilities and proceeded with the calibration using the standard AIPS amplitude, phase, and bandpass calibration routines vlacalib, vlaclcal, and bpass. We mapped and cleaned cubes for each IF pair using the task imagr and then used these cubes to identify the extent of the line-free channels. We then went back to the UV data and subtracted the continuum emission. The continuumsubtracted, calibrated UV data set was the basis for our final maps.

\section{NGC 6764}

Different authors have classified NGC 6764 [SB(s)bc] as a Seyfert 2 galaxy (Rubin, Thonnard, \& Ford 1975) and as a low-ionization emission-line region (Eckart et al. 1991). It is a strongly barred galaxy with a compact, nuclear knot bright in both optical emission lines (Eckart et al. 1991) and the radio continuum (Baum et al. 1993). NGC 6764 also contains an $\sim 4 \mathrm{kpc}$ radio continuum plume indicative of a strong galactic wind (Baum et al. 1993). Eckart et al. (1996) studied the optical, near-IR, and millimeter properties of the nucleus of NGC 6764 and concluded that it is under-

${ }^{1}$ The VLA is part of the National Radio Astronomy Observatory, which is operated by Associated Universities, Inc., under a cooperative agreement with the National Science Foundation.

TABLE 2

VLA ObSERVATIONAL PARAMETERS

\begin{tabular}{|c|c|c|}
\hline Parameter & NGC 6764 & Mrk 1126 \\
\hline Observation date $\ldots \ldots \ldots \ldots \ldots \ldots$ & 1995 Apr 1 & 1995 Apr 1 \\
\hline Configuration .................... & $\mathrm{D}$ & $\mathrm{D}$ \\
\hline Time on source $(\mathrm{hr})$. & 1.7 & 2.7 \\
\hline 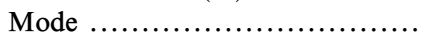 & $4 \mathrm{IF}$ & $4 \mathrm{IF}$ \\
\hline Channel separation $\left(\mathrm{km} \mathrm{s}^{-1}\right) \ldots \ldots$ & 21.1 & 21.1 \\
\hline Synthesized beam $(\operatorname{arcmin}) \ldots . . . .$. & $57 \times 51$ & $62 \times 43$ \\
\hline
\end{tabular}

going an intense burst of star formation that is only a few times $10^{7}$ yr old.

We used the imagr task in AIPS to transform and clean the UV data. We cleaned several channels using a range of different weighting schemes before settling on a robustness value of 3 , where +5 is a pure natural weight and -5 is a pure uniform weight. The resulting synthesized beam has a FWHM of $57^{\prime \prime} \times 51^{\prime \prime}$. The final channel maps have an rms noise of $0.6 \mathrm{mJy}$ beam $^{-1}$, which corresponds to a $1 \sigma$ column density sensitivity of $5 \times 10^{18} \mathrm{~cm}^{-2}$.

\subsection{H I Distribution and Morphology}

We show the channel maps in Figure 1, where the contours are $6,10,20,40$, and $80 \sigma$ and the peak column density is $1.8 \times 10^{20} \mathrm{~cm}^{-2}$. At both the high- and low-velocity extremes of the galaxy, there are modest extensions of $\mathrm{H}$ i to the north (at $2538 \mathrm{~km} \mathrm{~s}^{-1}$ ) and south (at $2287 \mathrm{~km} \mathrm{~s}^{-1}$ ), while the rest of the cube reflects a rotating disk with a modest $\mathrm{H}$ I depression at central velocities. To generate moment maps for NGC 6764, we blanked each channel of the cleaned cube at the $3^{\prime \prime}$ level and proceeded with the moment analysis. We show the isovelocity contours overlaid on the total $\mathrm{H}$ I column density map in Figure 2, a comparison of the $\mathrm{H} \mathrm{I}$ and stellar distributions in Figure 3, and the total $\mathrm{H}$ I profile in Figure 4. The total $\mathrm{H}$ I mass is $6.9 \times 10^{9} M_{\odot}$, down to a column density of $1.5 \times 10^{19}$ $\mathrm{cm}^{-2}$.

As is evident in the channel maps, the $\mathrm{H}_{\mathrm{I}}$ in NGC 6764 is concentrated in two clumps on either side of a modest central depression. Comparing the $\mathrm{H}$ I and stellar distributions in Figure 3, we see that the two $\mathrm{H}$ I clumps are coincident with the ends of the stellar bar with the central H I depression coincident with the high surface brightness bulge of the galaxy. The central depression is also coincident with the radio continuum plume identified by Baum et al. (1993) and a continuum source with a peak flux density of $102 \mathrm{mJy}$ (from the NASA/IPAC Extragalactic Database [NED]). In fact, the major axis of the radio plume parallels the straight isovelocity contours evident in Figure 2 in the inner part of NGC 6764. Given the presence of a continuum source associated with the nucleus of the galaxy, it is possible that the observed depression in the $\mathrm{H}$ I column density in the center of the galaxy reflects $\mathrm{H}_{\mathrm{I}}$ absorption against the 102 mJy source. Our data is not of sufficient resolution to thoroughly test this proposition.

To quantitatively characterize the $\mathrm{H}$ I morphology, we fitted a two-dimensional Gaussian to the outermost $\mathrm{H}$ I contour, which corresponds to a surface density of $1 M_{\odot}$ $\mathrm{pc}^{-2}$, and derived semimajor and semiminor axes of $124^{\prime \prime}$ and $87^{\prime \prime}$, respectively. If the true $\mathrm{H}$ I distribution is approximately circular, these dimensions imply an inclination of 44.5. Using the optical axial ratio of 1.7 (NED) we derive an inclination of $36^{\circ}$. Assuming $H_{0}=65 \mathrm{~km} \mathrm{~s}^{-1} \mathrm{Mpc}^{-1}$ and taking the recessional velocity of NGC 6764 to be $2416 \mathrm{~km}$ $\mathrm{s}^{-1}$, we derive a projected $\mathrm{H}$ I diameter of $45 \mathrm{kpc}$. Because of the effects of beam-smearing, this is an upper limit to the $\mathrm{H}$ I extent of the galaxy. According to NED, the extent of the stellar distribution is 2.3 , corresponding to $25 \mathrm{kpc}$. This yields a $D_{\mathrm{H} \text { I }} / D_{\text {stellar }}$ ratio of 2.2 , consistent with that of most large, spiral galaxies (Broeils \& van Woerden 1994).

There are interesting variations in the position angle of the $\mathrm{H}$ I distribution in NGC 6764. Outside of the inner $3^{\prime}$, the position angle of the $\mathrm{H}$ I distribution shifts from $40^{\circ}$ to $58^{\circ}$. We can see this shift in the position angle in the form of 


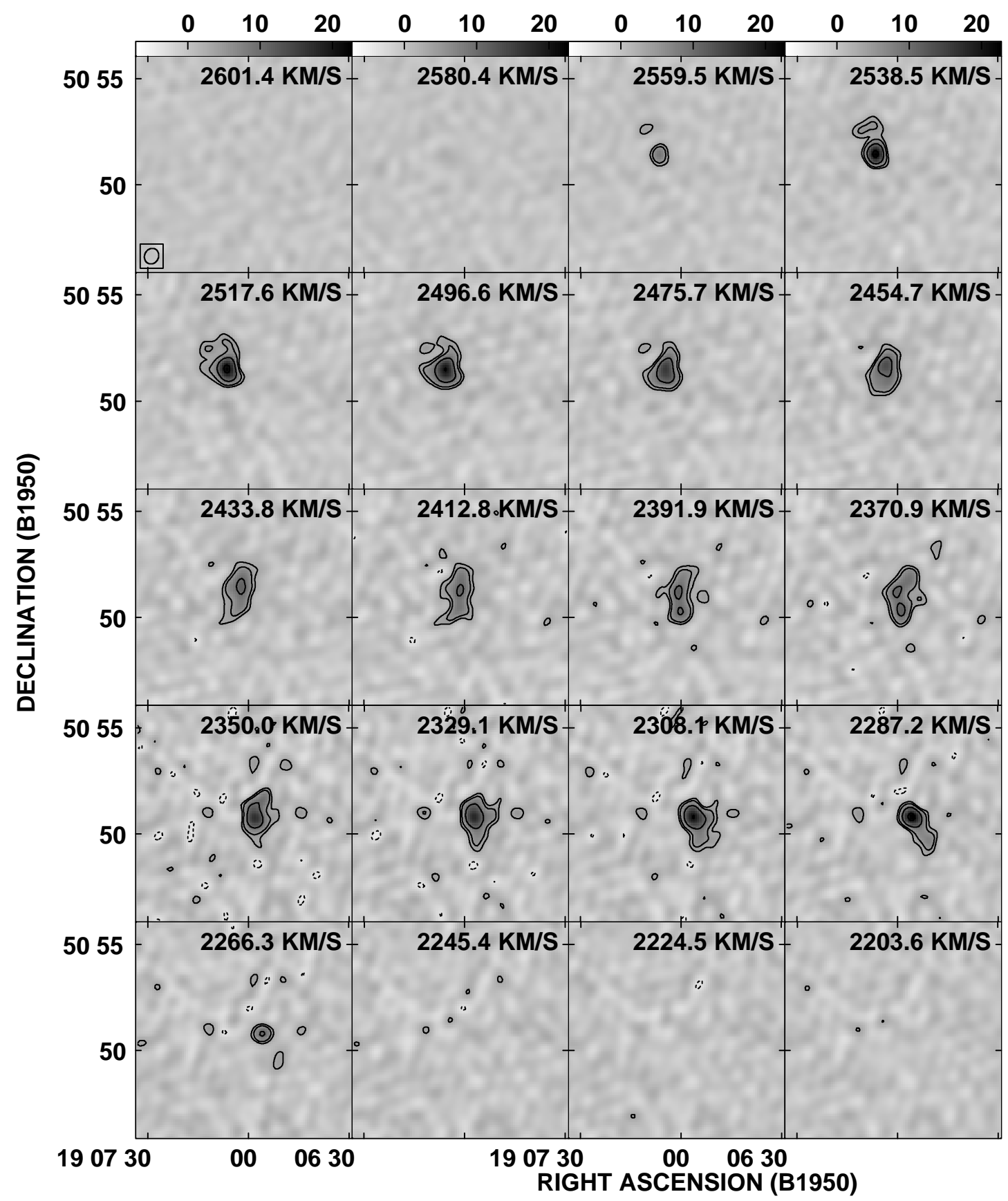

Fig. 1.- Channel maps for NGC 6764. The contours correspond to column densities of $-2 \times 10^{19}, 2 \times 10^{19}, 3.1 \times 10^{19}, 5.1 \times 10^{19}, 1.0 \times 10^{20}$, $2.0 \times 10^{20}$, and $4.0 \times 10^{20} \mathrm{~cm}^{-2}$. The rms noise is $5 \times 10^{18} \mathrm{~cm}^{-2}$. There are modest extensions of $\mathrm{H}$ i on the northern edge of the galaxy at $2538 \mathrm{~km} \mathrm{~s} \mathrm{~s}^{-1}$ and at the southern edge at $2287 \mathrm{~km} \mathrm{~s}^{-1}$. The beam $\left(57^{\prime \prime} \times 51^{\prime \prime}\right)$ is plotted in the lower left corner of the first panel.

the odd $\mathrm{H}$ I extensions at low $\left(2287 \mathrm{~km} \mathrm{~s}^{-1}\right)$ and high $(2538$ $\mathrm{km} \mathrm{s}^{-1}$ ) velocities in the channel maps in Figure 1 . Although the resolution is low, there is also a hint of a change in the position angle of the outermost gas in the isovelocity contours in Figure 2.

A $1 \sigma \mathrm{rms}$ noise of $0.6 \mathrm{mJy}$ beam $^{-1}$ corresponds to a $5 \sigma$ mass sensitivity of $2 \times 10^{7} M_{\odot}$. Our observations show that NGC 6764 does not have any gas-rich companions with $\mathrm{H}$ I masses greater than $2 \times 10^{7} M_{\odot}$ within a radius of $160 \mathrm{kpc}$, assuming a $30^{\prime} \mathrm{FWHM}$ primary beam. While there has been no systematic search for gas-rich companions around field galaxies, we can note that $48 \%$ of the optically identified satellites around field galaxies do lie within 160 kpc of their parent (Zaritsky et al. 1997), and all of the 12 companions found around nine "isolated" galaxies (out of a sample of 41 isolated galaxies) lie within $160 \mathrm{kpc}$ (Pisano 2001).

\subsection{Kinematics}

The velocity field, shown as isovelocity contours overlaid on the moment zero map in Figure 2, is unremarkable, except for what is possibly a warp at large radii. Over the region spanned by the optical bar, the isovelocity contours show the characteristic bent of noncircular motions and kinematic major and minor axes that are not perpendicular, confirming the existence of a bar in NGC 6764. Farther out 


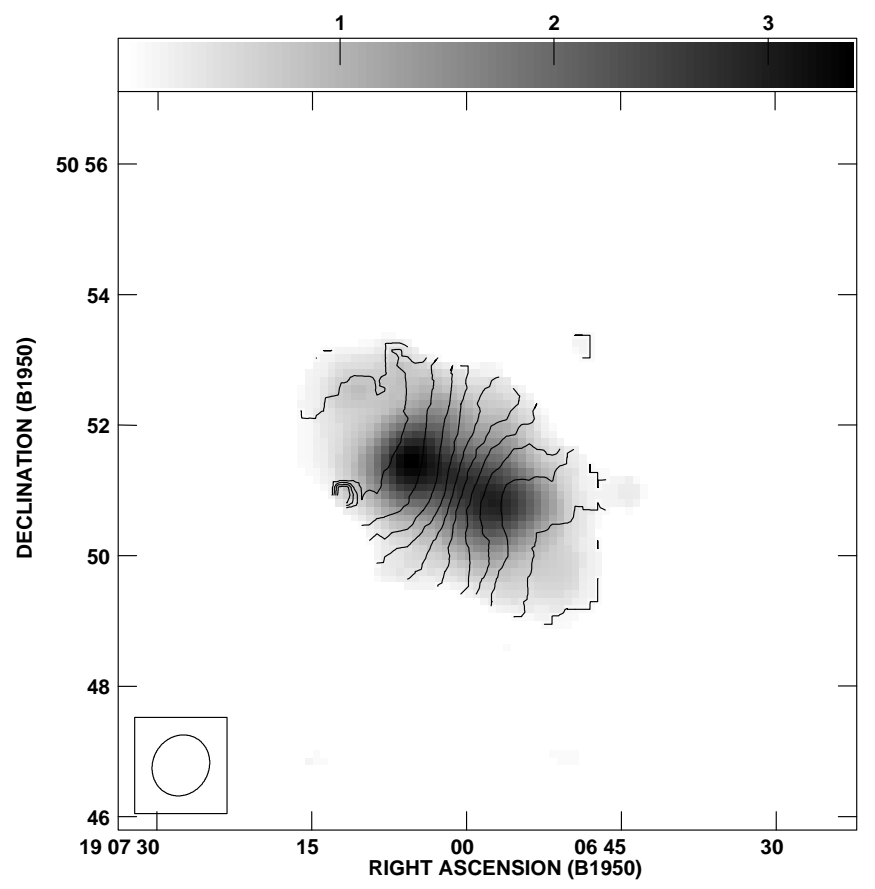

FIG. 2.-H I velocity field (contours) overlaid on the total column density map for NGC 6764. The maximum column density is $1.5 \times 10^{21}$ $\mathrm{cm}^{-2}$. The velocity contours begin at $2260 \mathrm{~km} \mathrm{~s}^{-1}$ with the closed contours at the southwestern edge and increase by $20 \mathrm{~km} \mathrm{~s}^{-1}$ per contour to the northeast. Same beam size as in Fig. 1.

we do detect a slight change in the position angle of the major axis, but such features are not uncommon among disk galaxies.

Analysis of the velocity field in Figure 2 and the $\mathrm{H}$ I profile in Figure 4 indicates that the solid-body part of the rotation curve extends over the inner $2^{\prime}$ of the galaxy. The

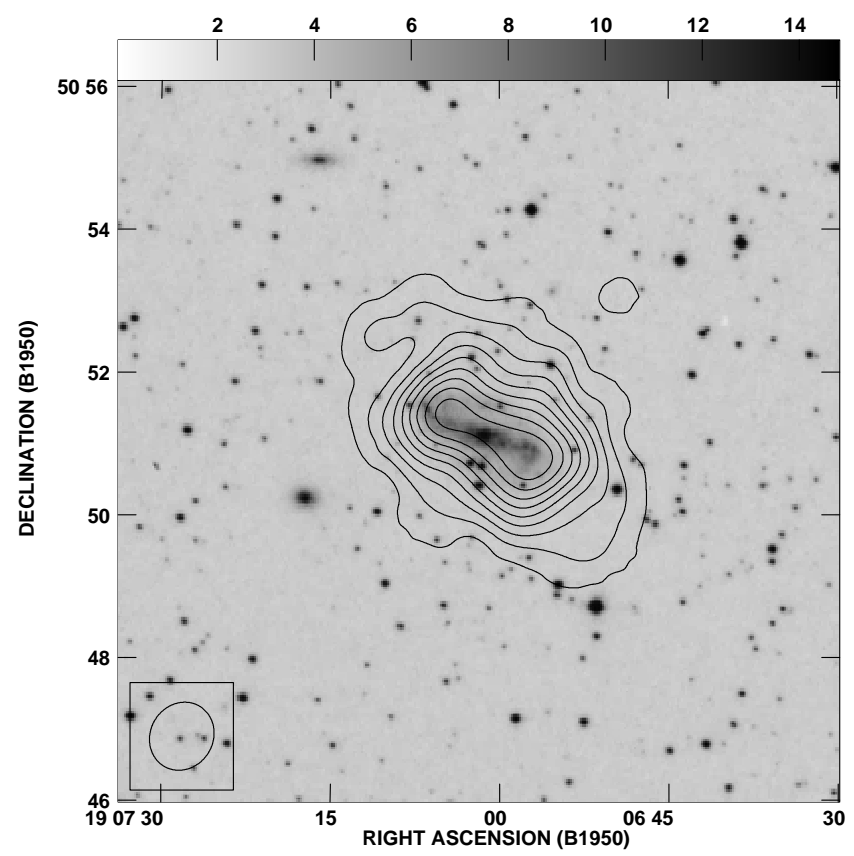

FIG. 3.-Total H I column density contours are plotted on a Digital Sky Survey image of NGC 6764. The projected H I diameter is $45 \mathrm{kpc}$, while the stellar extent is $25 \mathrm{kpc}$. Same beam size as in Fig. 1. The contours correspond to column densities of $1.3,2.6,3.9,5.2,6.5,7.8,8.1,9.4$, and $10.4 \times 10^{21} \mathrm{~cm}^{-2}$.

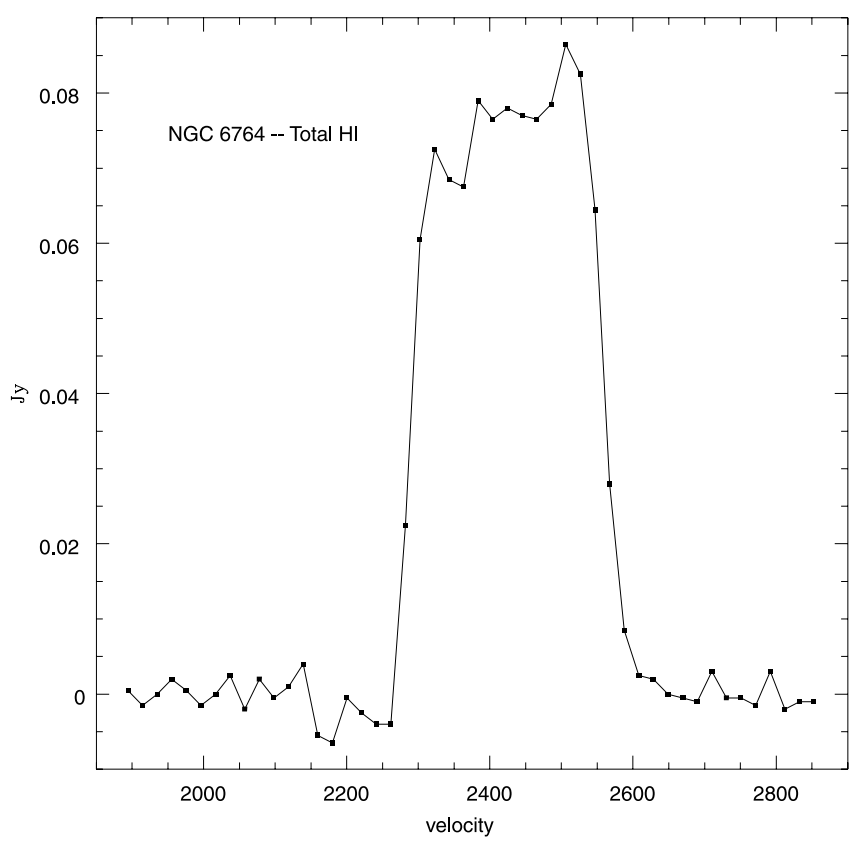

FIG. 4.- Integrated H I profile for NGC 6764 containing all emission down to a column density equivalent to $1 M_{\odot} \mathrm{pc}^{-2}$.

FWHM of the $\mathrm{H}$ I profile yields a projected rotational velocity of $115 \mathrm{~km} \mathrm{~s}^{-1}$ and a systematic velocity of $2424 \mathrm{~km}$ $\mathrm{s}^{-1}$. Correcting for an inclination of 44.5 , we derive a rotational velocity of $162 \mathrm{~km} \mathrm{~s}^{-1}$ for NGC 6764 . We derive a similar inclination calculating the maximum rotational velocity predicted by Persic \& Salucci (1991) using the $L_{B}$ listed in Table 1 and their equation (4). The resulting dynamical mass is between $1.9 \times 10^{11}$ and $1.3 \times 10^{11} M_{\odot}$.

\section{Mrk 1126}

Osterbrock \& Dahari (1983) classified Mrk 1126 (NGC 7450 ) as a narrow-line Seyfert 1.5 in a host with Hubble type SBa. Like NGC 6764, Mrk 1126 is a strongly barred galaxy with a bright, compact nucleus. Mrk 1126 is a likely member of a small group of galaxies that also includes NGC 7443 [SB(s)0], NGC 7444 (SB), and MCG -02-58017 (Garcia 1993). NGC 7443 and NGC 7444 are projected very close to one another on the sky but are separated by $\sim 600 \mathrm{~km} \mathrm{~s}^{-1}$ in velocity. The velocity difference between Mrk 1126 and NGC 7444 and NGC 7443 is $\pm 300 \mathrm{~km} \mathrm{~s}^{-1}$ (da Costa et al. 1998), but we did not detect either galaxy in our data cube, most likely because they are early-type galaxies.

As with NGC 6764, we used uvlin to subtract the continuum from Mrk 1126 in the UV plane. The final cube was cleaned using a robustness of 0 , yielding a synthesized beam of $62^{\prime \prime} \times 43^{\prime \prime}$. The $\mathrm{rms}$ noise was $0.6 \mathrm{mJy}^{-1}$ beam $^{-1}$, corresponding to a $1 \sigma$ column density of $3 \times 10^{18} \mathrm{~cm}^{-2}$.

\subsection{H I Distribution and Morphology}

The channel maps of Mrk 1126 in Figure 5 clearly show the presence of two galaxies at similar, but still distinct, velocities. Between 3633 and $3380 \mathrm{~km} \mathrm{~s}^{-1}$ we find MCG $-02-58-017$ to be barely resolved in the individual channel maps. Mrk 1126 lies between 3317 and $3085 \mathrm{~km} \mathrm{~s}^{-1}$, and the channel maps show that the emission at central velocities ( 3191 to $3170 \mathrm{~km} \mathrm{~s}^{-1}$ ) is concentrated in two clumps. At velocities of $3149-3086 \mathrm{~km} \mathrm{~s}^{-1}$, there is a small appendage 


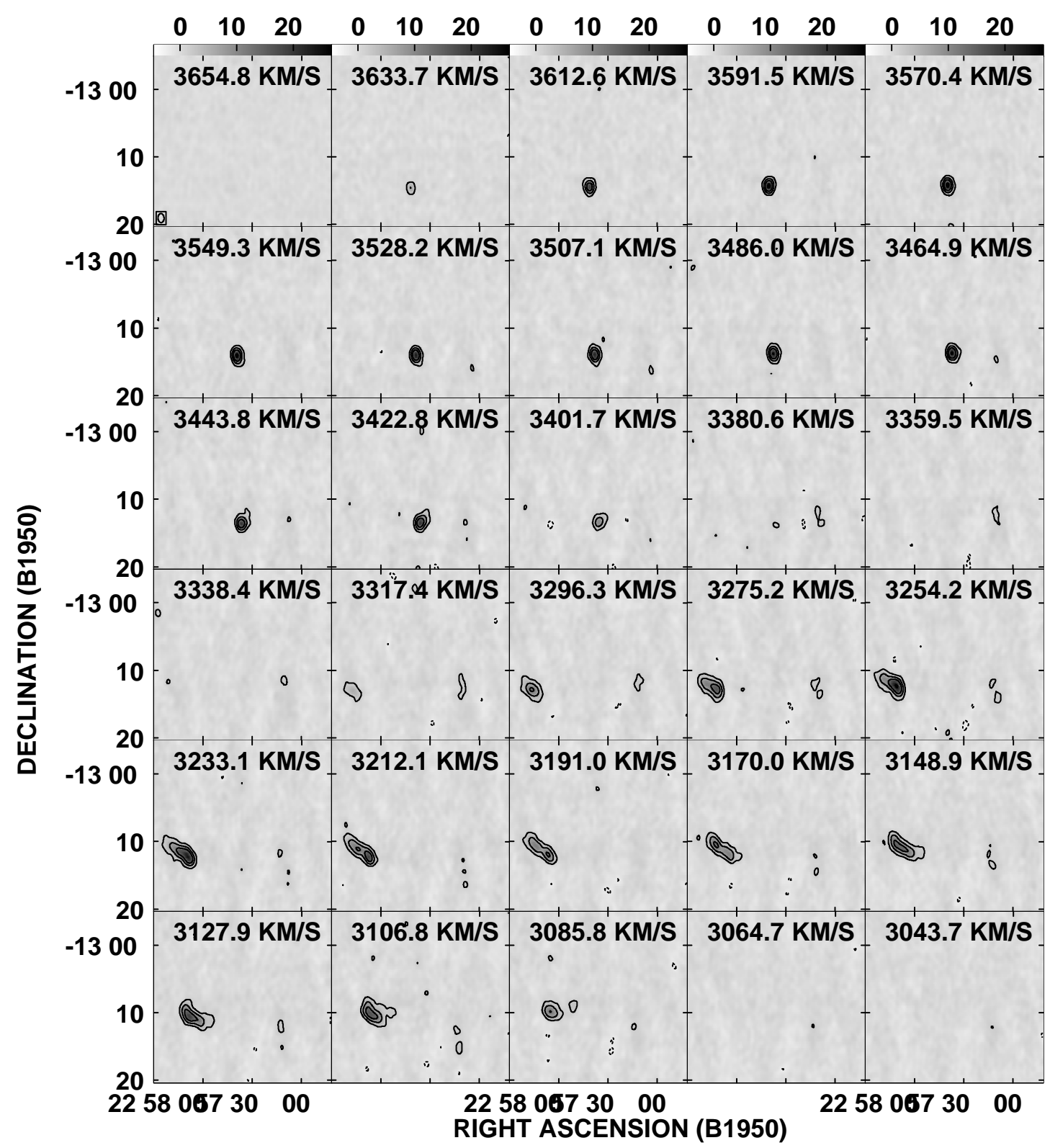

FIG. 5.-Channel maps for Mrk 1126 (between velocities of 3318 and $3108 \mathrm{~km} \mathrm{~s}^{-1}$ ) and MCG -02-58-017 (between velocities of 3612 and $3402 \mathrm{~km} \mathrm{~s}-1$ ). The rms noise corresponds to $3.0 \times 10^{18} \mathrm{~cm}^{-2}$. The contours are $1.1 \times 10^{19}, 1.5 \times 10^{19}, 3 \times 10^{19}, 4.5 \times 10^{19}, 7.5 \times 10^{19}, 1.5 \times 10^{20}, 3 \times 10^{20}$, and $4.5 \times 10^{20} \mathrm{~cm}^{-2}$. The synthesized beam of $62^{\prime \prime} \times 43^{\prime \prime}$ is plotted in the lower left corner of the first panel.

emanating from the western edge of the distribution of gas in Mrk 1126. We show the total H I map for Mrk 1126 and its companion in Figure 6. The subtle extension to the $\mathrm{H} \mathrm{I}$ disk of Mrk 1126 is more evident in this map than in the individual channels. The center-to-center separation between Mrk 1126 and MCG -02-58-017 is nearly $9^{\prime}$, corresponding to a projected separation of $100 \mathrm{kpc}$ at a distance of $49 \mathrm{Mpc}$.

One of the more notable features in the total $\mathrm{H}$ I map of Mrk 1126 and its companion is the extent of their disks. The extent of the $\mathrm{H}$ I contours in Figure 7, where we overlay the $\mathrm{H}$ I contours on top of a Digital Sky Survey (DSS) image, is somewhat misleading because we do have such a large beam. We fitted a two-dimensional Gaussian to the outermost H I contour of Mrk 1126 in Figure 6. The resulting semimajor axis of Mrk 1126 is 7.7. This is approximately 4 times larger than the optical extent of the galaxy of $1^{\prime} .8$
(NED). Because of the issue of beam-smearing this is an upper limit to the ratio of the $\mathrm{H}$ I diameter to the stellar diameter, but it does reinforce the idea that Mrk 1126 is a very gas-rich Seyfert. The H I distribution in MCG -02-58017 is quite centrally concentrated, but with a projected diameter of $55 \mathrm{kpc}$ (derived from a fit of an ellipse to the outermost $\mathrm{H}$ I contour) it, too, extends some 4 times beyond the optical diameter, $D_{25}$.

We plot the profiles for Mrk 1126, its small appendage (discussed below), MCG -02-58-017, and the entire system in Figure 8. Integrating the total $\mathrm{H} \mathrm{I}$ profiles and assuming a distance of $49 \mathrm{Mpc}$, we calculate an $\mathrm{H}$ i mass for the entire system, including the small clump, of $1.1 \times 10^{10} M_{\odot}$. We calculate $\mathrm{H}_{\mathrm{I}}$ masses of $7 \times 10^{9}$ and $4 \times 10^{9} M_{\odot}$ for Mrk 1126 and MCG - 02-58-017, respectively.

The hint of a low column density appendage to the $\mathrm{H} \mathrm{I}$ disk on the west side of Mrk 1126 and in the direction of its 


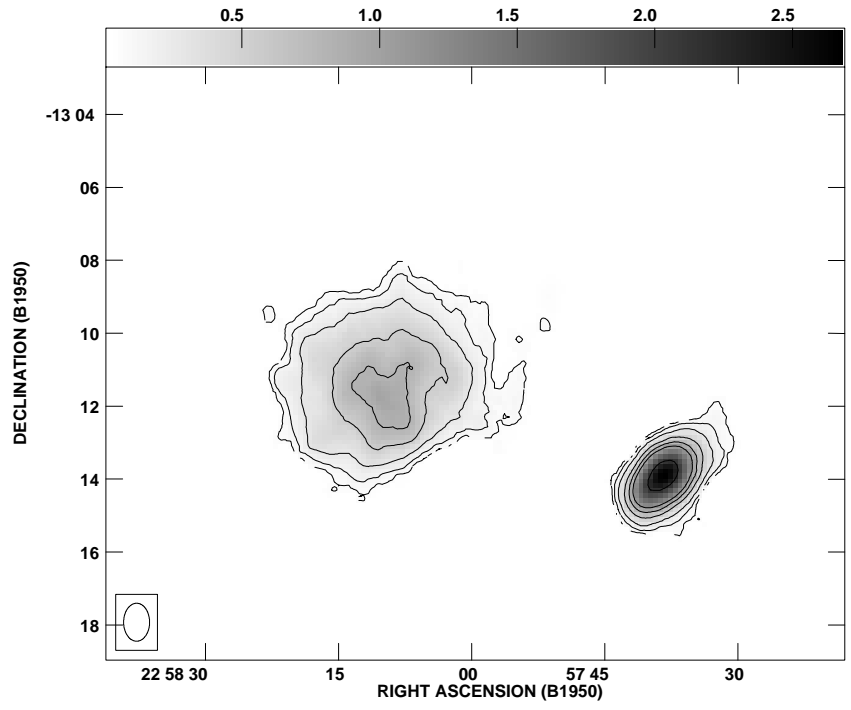

FIG. 6.-H I column density map for Mrk 1126 and MCG -02-58-017. The contours correspond to column densities of $1.7 \times 10^{19}, 3.4 \times 10^{19}$, $6.8 \times 10^{19}, 1.4 \times 10^{20}, 2.8 \times 10^{20}, 5.6 \times 10^{20}$, and $8.4 \times 10^{20} \mathrm{~cm}^{-2}$. Note the small extension off of the western edge of Mrk 1126. The beam is the same as in Fig. 5 and is plotted in the lower left corner.

companion is intriguing because it is comparable to $\mathrm{H} \mathrm{I}$ clouds detected in the vicinity of a number of other spirals. We are able to follow the appendage through four channels beginning at $3170 \mathrm{~km} \mathrm{~s}^{-1}$, but it is much more apparent in the total H I map. That we can trace the appendage smoothly over four channels suggests that it is real, but a conclusive determination would require more sensitive data at higher resolution. Without higher spatial resolution we also cannot tell if it is an integral part of the disk or a separate entity. If we treat it as a separate entity, and it does have a distinct $\mathrm{H}$ I profile as seen in Figure 8, and then estimate the amount of gas above a column density of $1.5 \times 10^{19} \mathrm{~cm}^{-2}$,

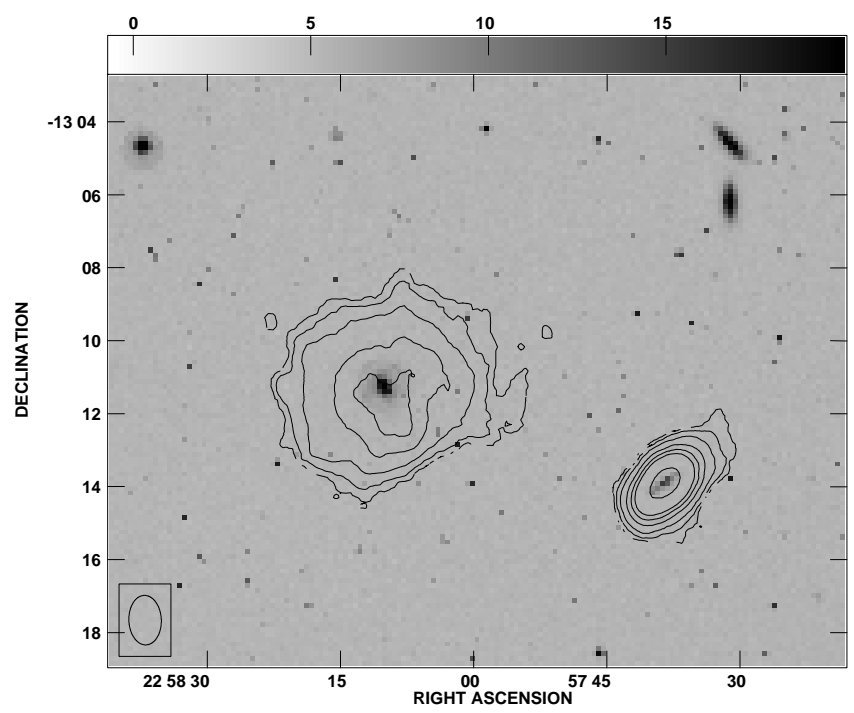

FIG. 7.-Contours of the $\mathrm{H}$ I column density, now plotted on a DSS image. Both galaxies have $\mathrm{H}$ I disks that extend $\sim 4$ times the optical diameter. The $\mathrm{H} \mathrm{I}$ beam size is the same as in Fig. 5 and is plotted in the lower left corner. The contours correspond to column densities of $1.7 \times 10^{19}, 3.4 \times 10^{19}, 6.8 \times 10^{19}, 1.4 \times 10^{20}, 2.8 \times 10^{20}, 5.6 \times 10^{20}$, and $8.4 \times 10^{20} \mathrm{~cm}^{-2}$. we derive a total $\mathrm{H}$ I mass of $2.1 \times 10^{8} M_{\odot}$. This is consistent with the $\mathrm{H}$ I masses of clouds detected near NGC 925 (Pisano et al. 1998), for example. Alternatively, and more likely, the appendage is the signature of a tidal interaction between Mrk 1126 and MCG -02-58-017. The fact that the velocity of the clump is a smooth continuation of the velocity field of Mrk 1126 supports this conclusion.

\subsection{Kinematics}

We overlay the velocity field on top of the total $\mathrm{H}$ I map in Figure 9. At this resolution the velocity field of Mrk 1126 is quite regular, although there is some evidence of what is most likely bar-induced twists in the isovelocity contours. The kinematics of MCG -02-58-017 appear to be dominated by solid-body rotation.

From the velocity field and the $\mathrm{H}$ I profile we derive a rotational velocity of $125 \mathrm{~km} \mathrm{~s}^{-1}$ (projected). Making a rough correction for an inclination of $55^{\circ}$ based on the ellipticity of the $\mathrm{H}$ I distribution, we derive a deprojected rotational velocity of $140 \mathrm{~km} \mathrm{~s}^{-1}$. This is a bit less than the $160 \mathrm{~km} \mathrm{~s}^{-1}$ we get applying the Persic \& Salucci (1991) universal rotation curve. We also note that the ratio of the optical axes is 1, suggesting that Mrk 1126 is being viewed face-on, which is clearly not the case. The huge $\mathrm{H}$ I disk has a measured radius of $41 \mathrm{kpc}$, yielding an estimate of the dynamical mass of $1.9 \times 10^{11} M_{\odot}$. Because we are using a relatively large beam, the actual $\mathrm{H}$ I radius is likely smaller than the one we have measured.

The rotation curves of both MCG - 02-58-017 and Mrk 1126 are best seen in a position-velocity (PV) diagram such as that shown in Figure 10. We rotated the cube clockwise by $32^{\circ}$ to align the major axes of both galaxies with the horizontal axis of the cube. We then summed this rotated cube along the minor axes of each galaxy to produce the PV diagrams seen in Figure 10. The rotation curve of MCG $-02-58-017$ is linear with little, if any, indication of flattening. Taking $V_{\text {rot }}$ to be $115 \mathrm{~km} \mathrm{~s}^{-1}$ and the $\mathrm{H}$ I radius to be $29 \mathrm{kpc}$, one can estimate the dynamical mass of MCG $-02-58-017$ to be close to $10^{11} M_{\odot}$. Keep in mind that because the rotation curve is still rising at the last measured point, and the inclination is unknown and hence uncorrected for, this estimate of the mass is a lower limit. From this perspective MCG -02-58-017 is very massive, gas-rich galaxy that still appears to be dominated by solid-body rotation. In the same PV diagram we also note that Mrk 1126 has a flat rotation curve almost to the full measured extent of its $\mathrm{H}$ I disk.

\section{ANALYSIS AND DISCUSSION}

An obvious question is whether the presence of MCG $-02-58-017$ is at all related to the nuclear activity in Mrk 1126. One measure of the strength of the interaction between Mrk 1126 and its neighbor is the Dahari parameter, $Q_{\mathrm{D}}$ (Dahari 1984), that has been used by Byrd, Sundelius, \& Valtonen (1987) to quantify the tidal triggering of Seyfert activity. $Q_{\mathrm{D}}$ is given by

$$
Q_{\mathrm{D}}=\left(M_{\text {neighbor }} / M_{\text {galaxy }}\right) /\left(R_{\text {sep }} / R_{\text {galaxy }}\right)^{3} .
$$

Here, the subscript "galaxy" refers to the primary galaxy. $R_{\text {sep }}$ is the apparent separation between the two systems and $M_{\text {neighbor }}$ is the mass of the neighbor. For the Mrk 1126 system we derive a $Q_{\mathrm{D}}$ of only 0.04 using the dynamical masses and the $\mathrm{H}$ I diameter of Mrk 1126. This is far less than the $Q_{\mathrm{D}} \sim 1$ needed to trigger nuclear activity (Byrd 

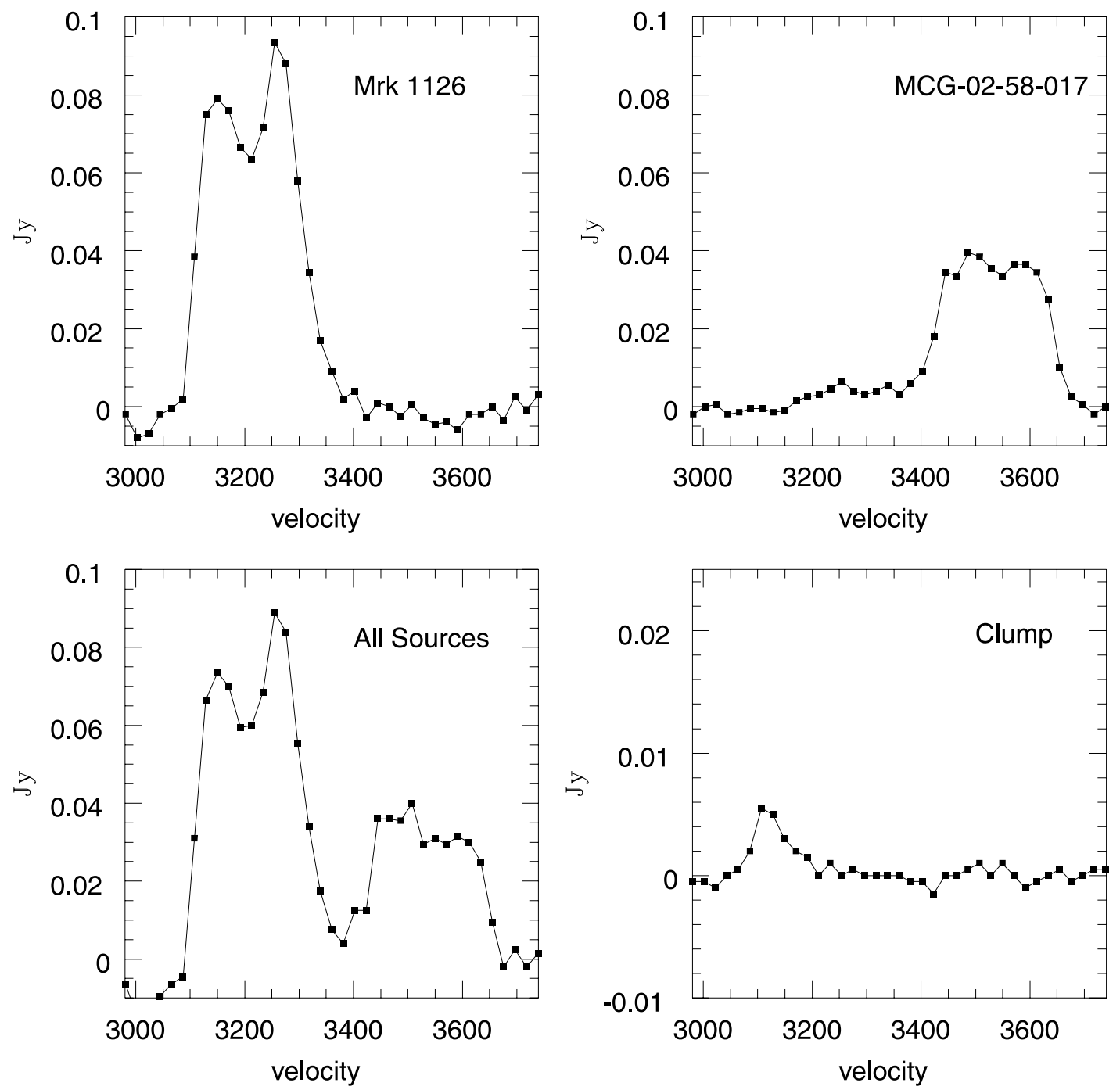

FIG. 8.-H I profiles for Mrk 1126 (upper left), MCG -02-58-017 (upper right), the total system (lower left), and the small gaseous appendage to Mrk 1126 (lower right).

et al. 1987). So even though Mrk 1126 does have a massive companion, it is extremely unlikely that MCG -02-58017 is triggering the nuclear activity in Mrk 1126. Other than the small extension discussed in the previous section, there is no evidence in the form of $\mathrm{H}$ I bridges or tails that Mrk 1126 has experienced any other recent interaction.

Another tracer of the impact of interactions is the relative asymmetry of the galaxies involved. One can approach this in a number of ways, including measuring the asymmetry in the H I profile (e.g., Richter \& Sancisi 1994; Haynes et al. 1998) or in the distribution of light in the optical disk (e.g., Conselice, Bershady, \& Jangren 2000). Kornreich et al. (2000) also use the difference between the rotation curve on either side of the dynamical center. Since our low angular resolution keeps us from accurately deriving a rotation curve, we will use the first two methods to determine the asymmetry of Mrk 1126 and its companion.

Using broadband optical images obtained with the Wisconsin-Indiana-Yale-NOAO $3.5 \mathrm{~m}$ telescope and following the technique pioneered by Conselice et al. (2000), we derived asymmetry values for Mrk 1126 and MCG $-02-58-017$ of $0.07 \pm 0.01$ and $0.08 \pm 0.06$, respectively. An asymmetry value of 0.07 is consistent with a galaxy whose stellar distribution is not significantly disturbed by an interaction or merger. What little asymmetry there is may simply be the result of star formation. The large error bar on the asymmetry value of MCG -02-58-017 leaves open the possibility that its stellar distribution is being slightly affected by the interaction. But in neither galaxy is the morphology of the stellar distribution dominated by the proximity of a neighbor.

The $\mathrm{H}$ I distributions of the two galaxies are more obviously asymmetric, particularly at the outermost contours. The asymmetry is also reflected in the $\mathrm{H}$ I profile for Mrk 1126. The ratio of the width of the profile at $50 \%$ of the peak to that at $20 \%$ of the peak is 0.77 , while the ratio of the areas under the two horns of the profile is 0.89 . The ratio of the peaks of the two horns is 0.82 . These numbers suggest that the $\mathrm{H}$ I distribution of Mrk 1126 is quite asymmetric, more so than the majority of the galaxies examined by Haynes et al. (1998). Interestingly, NGC 6764, a galaxy that 


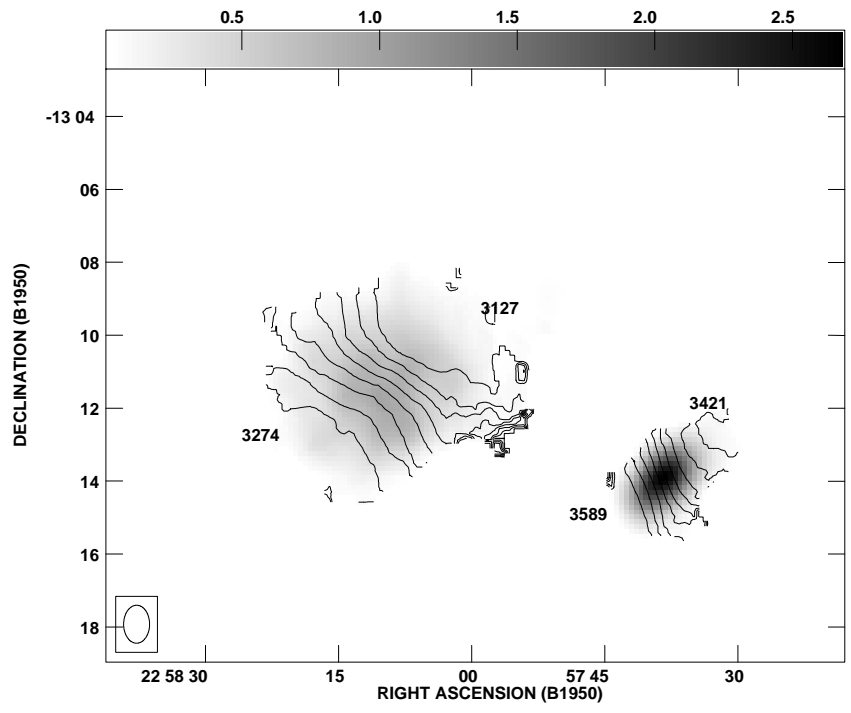

FIG. 9.-Velocity field of Mrk 1126 and MCG - 02-58-017 (contours) is overplotted on the $\mathrm{H}$ I column density map. For Mrk 1126, the isovelocity contours range from $106 \mathrm{~km} \mathrm{~s}^{-1}$ on the northwest side to $3274 \mathrm{~km} \mathrm{~s}^{-1}$ on the southeast side in steps of $21 \mathrm{~km} \mathrm{~s}^{-1}$. For MCG -02-58-017, the contours range from $3421 \mathrm{~km} \mathrm{~s}^{-1}$ on the northwest side to $3589 \mathrm{~km} \mathrm{~s}^{-1}$ on the southeast side. The beam size is the same as in Fig. 5 and is plotted in the lower left corner.

is apparently isolated, has similar asymmetries in its $\mathrm{H} \mathrm{I}$ profile: the ratio of the two peaks of the horns is 0.84 and the $W_{50} / W_{20}$ ratio is 0.82 .

We can conclude from this analysis that if Mrk 1126 and MCG - 02-58-017 are interacting, the effect is sufficiently strong to distort the outer $\mathrm{H}$ I distributions of the galaxies but has had negligible effect on either the distribution of gas or stars in the inner parts of the galaxies. It is, therefore, extremely unlikely that the nuclear activity is correlated with the interaction between Mrk 1126 and MCG -02-58017.

We cannot, however, rule out the possibility that some other external perturbation is responsible for the nuclear activity. Recent optical studies of the environments of Seyfert galaxies suggest that minor mergers, the accretion of small companion(s) amounting to $\sim 10 \%$ of the mass of the Seyfert, may play a role in triggering nuclear activity (De Robertis et al. 1998). Clearly, one motivation for this suggestion is the absence of evidence that Seyfert activity is tied to ongoing major interactions. If the conclusions of De Robertis et al. (1998) apply to either Mrk 1126 or NGC 6764 , then perhaps these galaxies have accreted a smaller gas cloud or dwarf companion. While the immediate environment of NGC 6764 is devoid of any companions with $\mathrm{H}$ I masses greater than $\sim 10^{7} M_{\odot}$, we have detected a low column density appendage to Mrk 1126 that could be interpreted as either a discrete cloud of gas or as a tidal feature.

Similar small clouds of $\mathrm{H}_{\mathrm{I}}$ have been detected around disk galaxies of all types, including dwarf irregulars like IC 10 (Wilcots \& Miller 1998), Magellanic spirals like NGC 4254 and NGC 4288 (Phookun et al. 1993; Wilcots et al. 1996), large Sc galaxies like NGC 628 (Kamphuis \& Briggs 1992), and, more recently, Seyfert galaxies like NGC 5506 (C. Mundell 2000, private communication). We now add Mrk 1126 to this growing list of galaxies. In some of these cases, and perhaps all, the clouds may be remnants of past tidal interactions, but it is difficult to ascertain their origin. Similarly, the role of small $\mathrm{H}$ I clouds in affecting the morphology and evolution of their host is not yet clear. The amount of material in the gaseous appendage as a percentage of the mass of Mrk 1126 is quite small (2.1\%). Any significant impact to Mrk 1126 would come only as a result of the accretion of this extra gas. With the low spatial and kinematic resolution of the data presented here, we cannot

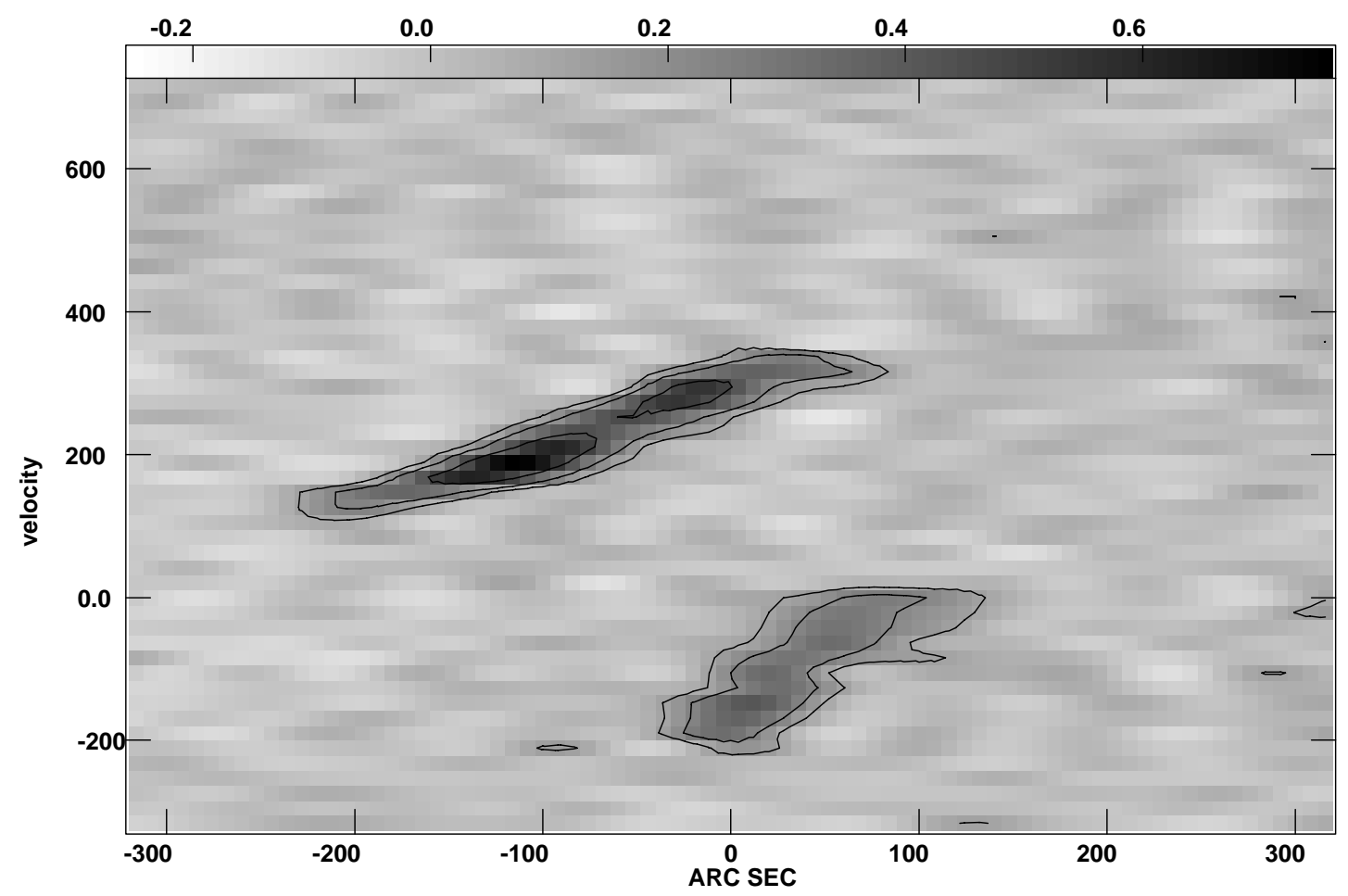

FIG. 10.-Position-velocity diagram obtained after rotating the cube clockwise by $32^{\circ}$ and summing along the minor axis of each galaxy. Both galaxies have very linear rotation curves, and while the one for Mrk 1126 flattens at large radii, the one for MCG -02-58-017 (upper left) does not. 
ascertain the origin or the fate of the small gaseous appendage to Mrk 1126.

We cannot rule out the possibility that NGC 6764 has recently accreted some amount of material. The distortions in the outer part of the $\mathrm{H}$ I distribution and the asymmetries of the $\mathrm{H}$ I profile are consistent with the presence of a warp and other weak external perturbation. As with Mrk 1126, we see no evidence that NGC 6764 has recently participated in a strong tidal interaction.

\section{CONCLUSIONS}

The initial goal of this study was to search the environments of a handful of Seyfert galaxies in order to find evidence for external triggers for the nuclear activity. The results are that NGC 6764 appears to be relatively isolated, and Mrk 1126 appears to be largely unaffected by the presence of a neighbor. Certainly, neither galaxy is currently involved in a strong interaction (although we cannot put constraints on past encounters). On the other hand, each galaxy shows some signs of having been the victim of a weak perturbation: the warped $\mathrm{H}$ I distribution and asymmetric profile of NGC 6764, and the presence of a small cloud of gas and the asymmetric profile of Mrk 1126. At this stage there is no way of determining whether these macro- scopic features are related to the nuclear activity. Of course, the nuclear activity could be fueled by strong bars, a trait both of these galaxies share.

The role of minor mergers needs to be investigated further. Although we see some evidence that Mrk 1126 and NGC 6764 may be currently accreting small amounts of gas, we lack the resolution necessary to thoroughly study this possibility. Our results echo a growing number of studies (e.g., De Robertis et al. 1998; Thean et al. 1997; Virani et al. 2000), and we can only conclude that there is no obvious relationship between nuclear activity in these two galaxies and their immediate environments.

We would like to thank Chris Conselice for his help in examining the optical asymmetries of the galaxies and Carole Mundell for sharing her recent results. E. M. W was supported by NSF grant AST 9691607. E. B. gratefully acknowledges support from the Consejo Nacional de Cencia y Technología under grant 27606-E. This research made use of the NASA/IPAC Extragalactic Database (NED), which is operated by the Jet Propulsion Laboratory, California Institute of Technology, under contract with the National Aeronautics and Space Administration.
Adams, T. F. 1977, ApJS, 33, 19

Baum, S. A., O'Dea, C. P., Dallacassa, D., de Bruyn, A. G., \& Pedlar, A. 1993, ApJ, 419, 553

Broeils, A., \& van Woerden, H. 1994, A\&AS, 107, 129

Byrd, G. G., Sundelius, B., \& Valtonen, M. 1987, A\&A, 171, 16

Conselice, C. J., Bershady, M. A., \& Jangren, A. 2000, ApJ, 529, 886

da Costa, L. N., et al. 1998, AJ, 116, 1

Dahari, O. 1984, AJ, 89, 966

De Robertis, M. M., Yee, H. K. C., \& Hayhoe, K. 1998, ApJ, 496, 93

Eckart, A., Cameron, M., Boller, Th., Krabbe, A., Blietz, M., Nakai, N., Wagner, S. J., \& Sternberg, A. 1996, ApJ, 472, 588

Eckart, A., Cameron, M., Jackson, J. M., Genzel, R., Harris, A. I., Wild, W., \& Zinnecker, H. 1991, ApJ, 372, 67

Fuentes-Williams, T., \& Stocke, J. T. 1988, AJ, 96, 1235

Garcia, A. M. 1993, A\&AS, 100, 47

Haynes, M. P., Jore, K. P., Barrett, E. A., Boreils, A. H., \& Murray, B. M. 2000, AJ, 120, 703

Haynes, M. P., van Zee, L., Hogg, D., Roberts, M. S., \& Maddalena, R. J. 1998, AJ, 115, 62

Hernquist, L., \& Mihos, J. C. 1995, ApJ, 448, 41

Kamphuis, J., \& Briggs, F. 1992, A\&A, 253, 335

\section{REFERENCES}

Kornreich, D. A., Haynes, M. P., Lovelace, R. V. E., \& van Zee, L. 2000, AJ, 120, 139

Laurikainen, E., \& Salo, H. 1995, A\&A, 293, 683

Mundell, C. G., Pedlar, A., Axon, D. J., Meaburn, J., \& Unger, S. W. 1995, MNRAS, 277,641

Osterbrock, D. E., \& Dahari, O. 1983, ApJ, 273, 478

Persic, M., \& Salucci, P. 1991, ApJ, 368, 60

Phookun, B., Vogel, S. N., \& Mundy, L. G. 1993, ApJ, 418, 113

Pisano, D. J. 2001, Ph.D. thesis, Univ. of Wisconsin, Madison

Pisano, D. J., Wilcots, E. M., \& Elmegreen, B. G. 1998, AJ, 115, 975

Richter, O.-G., \& Sancisi, R. 1994, A\&A, 290, L9

Rubin, V. C., Thonnard, N., \& Ford, W. K., Jr. 1975, ApJ, 199, 31

Simkin, S. M., Su, H.-J., van Gorkom, J., \& Hibbard, J. 1987, Science, 235, 1367

Thean, A. H. C., Mundell, C. G., Pedlar, A., \& Nicholson, R. A. 1997, MNRAS, 290,15

Virani, S. N., de Robertis, M. M., \& VanDalfsen, M. L. 2000, AJ, 120, 1739

Wilcots, E. M., Lehman, C. \& Miller, B. 1996, AJ, 111, 1575

Wilcots, E. M., \& Miller, B. W. 1998, AJ, 116, 2363

Zaritsky, D., Smith, R., Frenk, C., \& White, S. D. M. 1997, ApJ, 478, 39 